\title{
Interferência de artefatos na detecção de fraturas radiculares verticais em dentes bovinos com núcleo metálico fundido em imagens tomográficas de feixe cônico
}

\author{
- Marjorie Fonseca da Cunha Departamento de Radiologia Dento-Maxilo-Facial, Faculdade de Odontologia de \\ São Leopoldo Mandic, Campinas, SP, Brasil • Francine Kulh Panzarella Departamento de Radiologia Dento- \\ Maxilo-Facial, Faculdade de Odontologia de São Leopoldo Mandic, Campinas, SP, Brasil • Gonzalo André \\ Montesinos Departamento de Estomatologia, Faculdade de Odontologia, Universidade de São Paulo, São Paulo, \\ SP, Brasil • Juliane Pirágine Araujo Departamento de Estomatologia, Faculdade de Odontologia, Universidade de \\ São Paulo, São Paulo, SP, Brasil • Ricardo Raitz Departamento de Radiologia Dento-Maxilo-Facial, Faculdade de \\ Odontologia de São Leopoldo Mandic, Campinas, SP, Brasil
}

RESUMO | Objetivos: Avaliar a interferência de artefatos (beamhardening) na detecção de fratura radicular vertical por meio da tomografia computadorizada de feixe cônico, bem como a sensibilidade, a especificidade e a acurácia. Materiais e métodos: Foram utilizadas 15 raízes de incisivos inferiores bovinos que, após a realização do tratamento endodôntico e desobturação de $10 \mathrm{~mm}$, foram reabilitadas com núcleo metálico fundido, cuja cimentação foi efetuada com cimento de fosfato de zinco. Os exames de tomografia computadorizada de feixe cônico foram realizados antes (To) e após (T1) a indução das fraturas, as quais foram avaliadas por dois examinadores, a fim de identificar a presença e a localização das fraturas radiculares, bem como a localização do beamhardening e sua interferência na detecção das fraturas radiculares verticais, utilizando o filtro Angio_Sharpen High $5 \mathrm{X}_{5}$. Resultados: O beamhardening foi observado em $100 \%$ das raízes e foi visualizado com mais frequência no terço radicular médio. Em ambos os momentos de avaliação (To e T1), os locais de beamhardening eram proporcionalmente os mesmos que os locais das fraturas, gerando diagnósticos positivos de fratura mesmo quando elas não existiam. Conclusões: A tomografia computadorizada de feixe cônico apresentou $78 \%$ de sensibilidade, $63 \%$ de especificidade e $68 \%$ de acurácia, demonstrando ser apenas um método auxiliar no diagnóstico de fraturas radiculares em dentes reabilitados com núcleo metálico fundido.

DESCRITORES | Artefatos; Tomografia Computadorizada Cone-beam; Fratura Dentária.

ABSTRACT | Interference of artifacts on the detection of vertical radicular fractures on bovine's teeth with metallic nucleus on cone beam computed tomography - Objectives: To evaluate the interference of artefacts (beamhardening) in the detection of vertical root fractures using the cone beam computed tomography and to measure its sensitivity, specificity and accuracy. Materials and Methods: 15 inferior incisive bovine roots were used after endodontic treatment and desobturation of 10 $\mathrm{mm}$ on each one. They were rehabilitated with a metallic nucleus, and cementation with zinc phosphate was made. The cone beam computed tomography exams were made before (To) and after (T1) the induction of fractures, which were evaluated by two independent examiners, aimed at evaluating the presence and localization of the dental root fractures, as well as the localization of beamhardening interference in the detection of vertical root fractures, using a Angio Sharpen High $5 \mathrm{X}_{5}$ filter. Results: The presence of beamhardening were detected in $100 \%$ of the roots, which were more frequently observed on the middle third of the roots. In both moments of the evaluation (To and T1), the localization of beamhardening were proportionally the same as those of local fractures, given a positive diagnoses of fracture even if they didn't exist. Conclusion: Cone beam computed tomography showed $78 \%$ of sensitivity, $63 \%$ of specificity and $68 \%$ of accuracy, showing that cone beam computed tomography is only an auxiliary method for diagnosis of dental root fractures in teeth treated with metallic nucleus.

DESCRIPTORS | Artifacts; Cone beam Computed Tomography; Tooth Fractures.

AUTOR CORRESPONDENTE Medicina e Odontologia São Leopoldo Mandic • Rua Heitor Penteado, 1832, 101A São Paulo, SP, Brasil • 05438-300 E-mail: ricardoraitz@ig.com.br 


\section{INTRODUÇÃO}

As fraturas radiculares são mais encontradas nos dentes com tratamento endodôntico convencional do que nos dentes não tratados endodonticamente., ${ }^{1,2,3}$ Ao comparar-se a incidência de fraturas radiculares incompletas que ocorrem em dentes com ou sem pinos radiculares, os mesmos podem causar mais fraturas do que o próprio tratamento endodôntico., ${ }^{1,2}$

A modalidade mais comum para o diagnóstico de fraturas radiculares verticais (FRV) na rotina clínica é a radiografia intraoral convencional e digital (2D). As FRV são normalmente orientadas no sentido vestíbulo-lingual, o que dificulta sua detecção nas radiografias periapicais, especialmente no estágio inicial da fratura. ${ }^{3-5}$ Por outro lado, imagens tridimensionais, como aquelas obtidas por exames de tomografia computadorizada de feixe cônico (TCFC), podem minimizar a limitação imposta. ${ }^{3,6} \mathrm{~A}$ natureza tridimensional da TCFC permite, em tese, a visualização da linha de fratura de múltiplos ângulos e de diferentes orientações, em cortes finos e com alto contraste.?

A presença de retentores intrarradiculares e agentes cimentantes hiperdensos podem gerar artefatos sob a forma de estrias horizontais ou raios, denominados beam hardening, que pode ser um entrave ao diagnóstico das fraturas radiculares. ${ }^{3.5,8,9}$ A interferência do artefato metálico causado pelos materiais obturadores e retentores intrarradiculares pode ser confundida com linhas de fraturas, levando a um falso diagnóstico.3.5,5,9,10,11

O objetivo deste estudo foi avaliar a interferência de artefatos (beam hardening) na detecção de FRV por meio de TCFC, bem como a sensibilidade, a especificidade e a acurácia.

\section{MATERIAS E MÉTODOS}

Este trabalho foi aprovado pelo Comitê de Ética da Faculdade de Odontologia e Centro de Pesquisa Odontológica São Leopoldo Mandic sob o protocolo 2010/o388.

Foram selecionados 15 incisivos inferiores bovinos com comprimentos radiculares semelhantes. Seguindo a mesma metodologia utilizada por Soares et al., ${ }^{12}$ Os 15 dentes foram limpos com lâminas de bisturi, polidos com pedra-pomes, utilizando-se escova de Robinson em baixa rotação, e mantidos em solução de timol a $0,1 \%$. Os dentes foram seccionados em cortadeira metalográfica de precisão (Isomet 1000, Buehler, Lake Bluf, IL, EUA) de modo que todas as raízes possuíssem $15 \mathrm{~mm}$ de comprimento. As raízes foram inspecionadas em lupa estereoscópica (Eikonal EK3ST, São Paulo, SP, Brasil) para assegurar a ausência de trincas e fraturas, e posteriormente foram submetidas ao tratamento endodôntico por meio do preparo químico-mecânico, de acordo com a técnica movimento rotatório alternado, ${ }^{13}$ com confecção de batente apical e recuos escalonados até a lima $\mathrm{K}$ \# 50 (DentsplyMaillefer, Ballaigues, Suiça). A irrigação foi feita com solução aquosa de hipoclorito de sódio a 1\% (Asfer Ind. Quimica, São Caetano do Sul, SP, Brasil). Os condutos radiculares foram secos com cones de papel absorventes (Dentsply, Petropolis, RJ, Brasil) e obturados pela técnica de condensação lateral com cones de guta percha (Dentsply, Petropolis, RJ, Brasil), utilizando-se o cimento Sealer 26 (Dentsply, Petropolis, RJ, Brasil), manipulado conforme as instruções do fabricante e inserido com o auxílio de espaçadores digitais e condensadores verticais de Paiva. A partir dessa etapa, as raízes foram acondicionadas em ambiente com $100 \%$ de umidade relativa. ${ }^{12}$

Com auxílio de calcadores Paiva (SS White/ Duflex, Rio de Janeiro, RJ, Brasil) aquecidos, as raízes foram desobturadas em $10 \mathrm{~mm}$, preservandose cinco $\mathrm{mm}$ de guta-percha no ápice, e procedeu-se à preparação para o núcleo metálico fundido (NMF). Para tal, foram empregadas brocas Largo $\mathrm{n}^{0} 1$ a 5 (Angelus Ind. Prod. Odontológicos, Londrina, PR, Brasil) aplicadas sequencialmente, sem irrigação, montadas com limitadores travados a $10 \mathrm{~mm}$ de sua ponta ativa, posicionada no sentido do longo eixo da raiz. Após isolamento do conduto radicular com vaselina sólida (Ind. Farm. Rioquimíca, São José do Rio Preto, Brasil), foi realizada uma impressão do 
canal e, também, confeccionado o NMF, com resina acrílica auto polimerizável (Duralay, PoliDental Ind. Com., Cotia, SP, Brasil), de forma a padronizar todas suas extensões e términos. Na superfície lingual do molde assim obtido, foi confeccionado um nicho com uma ponta diamantada esférica $\mathrm{n}^{0} 1016$ HL (KG Sorensen Ind. Com., Barueri, SP, Brasil), acompanhando o longo eixo da raiz, com a finalidade de acomodar a ponta da máquina de ensaio universal durante a indução da fratura radicular. O molde em Duralay foi fundido em liga de níquel-cromo-titânio (Fit CastTitanium, Talladium do Brasil, Curitiba, $\mathrm{PR}$, Brasil). Os NMF foram submetidos à limpeza e desinfecção com álcool 70\% (Jalles Machado, Goianésia, Go, Brasil) por 30 segundos. A seguir, foram lavados em água corrente por um minuto e secos com jatos de ar. Para a cimentação do NMF utilizou-se cimento de fosfato de zinco (SS White, Rio de Janeiro, RJ, Brasil), cuja manipulação foi conduzida de acordo com as instruções do fabricante. Em seguida, o material foi inserido no conduto radicular preparado, com o auxílio de propulsor de Lentulo (DentsplyMaillefer, Ballaigues, Suiça).

O cimento foi aplicado na superfície do NMF e posicionado no conduto, sendo este mantido sob carga digital por cinco minutos, após os quais, foram removidos os excessos de cimento com auxílio de sonda exploratória. As raízes foram mergulhadas em cera sete (DentBras, Pirassununga, SP, Brasil), fundida até a região do colo, de maneira a formar uma camada de 0,5 $\mathrm{mm}$ de espessura. As raízes foram, então, embutidas em tubos de policloreto de vinila (PVC) com resina de poliéster (Polydyne, Hutchinson do Brasil, Taboão da Serra, SP, Brasil), para simular o suporte ósseo. A cera foi removida e o espaço correspondente preenchido com poliéster (Impregum F, 3M Espe, Saint Paul, MN, EUA), visando a simulação do ligamento periodontal e a reprodução do movimento do elemento dental no interior do alvéolo. Ao conjunto assim obtido atribuiuse a designação "corpo de prova" ou "espécime”.
Os espécimes foram posicionados e fixados em um dispositivo de aço-inoxidável, com superfície inclinada em um ângulo de $45^{\circ}$, objetivando a reprodução da inclinação formada pelos prolongamentos dos longos eixos dentários de incisivos superiores com seus antagonistas. Toda a metodologia para a indução de fraturas, utilizada neste trabalho, seguiu o experimento de Soares et al. ${ }^{12}$

O dispositivo foi posicionado em uma máquina de ensaios universal (EMIC DL 2000, EMIC Ind. Com., São José dos Pinhais, PR, Brasil), com célula carga de $2 \mathrm{kN}$ (200 kgF) e o corpo de prova submetido ao carregamento compressivo, aplicado com velocidade de $2 \mathrm{~mm} / \mathrm{min}$, utilizando-se uma ponta com $3 \mathrm{~mm}$ de diâmetro, até que se verificasse, graficamente ou pelo ruído característico (o que ocorresse primeiro), a fratura da raiz dentária. Os NMF foram reposicionados no conduto radicular, sem pressão, antes da tomada tomográfica final.

Os corpos de prova foram submetidos a duas tomadas tomográficas; To (no momento inicial), antes de serem submetidos às fraturas e $\mathrm{T} 1$ (momento final), após passarem pela máquina de fraturas. Após terem sido feitas as exposições tomográficas finais (T1), a constatação das fraturas foi realizada por meio de inspeção direta ou em lupa estereoscópica da superfície radicular de cada corpo de prova. Foi empregado grafite pulverizado, com finalidade de se visualizar a linha de fratura. A comprovação da fratura por esses métodos representou o diagnóstico "verdade" para verificar a validade, por meio das medidas de sensibilidade e especificidade dos exames radiográficos e tomográficos na detecção de fraturas radiculares. Apesar dos 15 corpos de provas terem sido submetidos à fratura, apenas 10 apresentavam presença real de fratura após a análise. As imagens tomográficas foram adquiridas por intermédio do tomógrafo computadorizado i-AT,120 KVp (kilovoltagem), $5 \mathrm{~mA}$ (miliamperagem); (ImagingSciences, Hatfield, PA, EUA), na Clínica de Radiologia Odontológica do Instituto e Centro de 
Pesquisas Odontológicas (CPO) São Leopoldo Mandic, local em que os corpos de prova foram escaneados utilizando o protocolo de aquisição de 40 segundos, com field of view (FOV) de $13 \mathrm{~cm}$ e com voxel de 0,25 mm. Foi realizada a reconstrução primária com máxima resolução e, na sequência, a reconstrução secundária, com cortes de 1,0 mm de espessura, obtendo-se, assim, imagens axiais, sagitais, coronais e parassagitais das raízes. Para avaliação das imagens, foi utilizado o filtro Angio Sharpen High $5 \times 5$.

Dois especialistas em radiologia, com mais de oito anos de experiência na avaliação de imagens tomográficas, previamente calibrados em um estudo piloto, analisaram os exames tomográficos obtidos (To e T1) seguindo uma ordem pré-estabelecida para a visualização das imagens, a fim de identificar a presença ou ausência de fratura radicular. Também foi anotada a localização da fratura e a existência ou não do artefato beam hardening (hipodenso) e sua localização, assinalando apenas uma alternativa para cada quesito perguntado. Os avaliadores receberam as orientações escritas, por extenso, de como deveria ser realizado o preenchimento das planilhas de avaliação. Os observadores desconheciam a ordem dos corpos de provas fraturados e não-fraturados e avaliaram as imagens de cada corpo de prova primeiramente nas direções axiais, sagitais e coronais. Permanecendo a dúvida quanto a presença de fratura, poderia ser utilizado o corte parassagital. As avaliações de todos os dentes ocorreram em dois tempos diferentes com intervalos de 15 dias. Não houve restrição de tempo para avaliação tomográfica e os avaliadores tiveram livre acesso às ferramentas de manipulação de imagem do software.

As imagens tomográficas foram adquiridas antes (To) e após a indução das fraturas radiculares (T1). Sendo assim, os 15 dentes foram avaliados em To e em T1 (resultando em 30 imagens tomográficas). Como cada examinador avaliou as imagens com intervalo de 15 dias nos dois momentos, cada avaliador observou um total de 60 imagens.
Os quesitos tomográficos avaliados foram: a) a presença ou ausência de FRV; e b) a presença do artefato beam hardening e sua localização.

Para a avaliação das imagens tomográficas foi utilizado um monitor de tela de cristal líquido (LCD em inglês) tela plana de 17" (LG modelo 5000:1), com resolução de 1280 x 1024 pixels e máxima qualidade de cor (12 bits) e o software dedicado ao tomógrafo (XoranCAT, version 3.1.62; Xoran Technologies, Ann Arbor, MI, EUA), aplicando-se o filtro Angio_Sharpen_ high 5x5, além de ajustes de brilho e contraste.

Para verificação da presença de fratura e se os locais de fratura coincidiram com o local do beam hardening foi utilizado o teste do Qui-Quadrado para amostras independentes. Os resultados desse teste estatístico mostraram, ao nível de $5 \%$ de significância, que não foram identificadas diferenças relevantes entre os avaliadores.

Para mensurar a concordância inter-examinadores quanto à presença de fraturas entre os momentos (To e T1), foi utilizado o teste estatístico Kappa ao nível de 5\% de significância. A validade dos exames tomográficos para detecção de fraturas foi verificada por meio das medidas de sensibilidade e especificidade.

\section{RESULTADOS}

O resultado do teste Kappa mostrou que a concordância inter-examinadores foi considerada moderada, tendo em vista que seu valor foi 0,552 , sendo estatisticamente significativa ( $\mathrm{p}$-valor $<0,05)$.

Das 15 raízes avaliadas, foram constatadas fraturas reais (diagnóstico "verdade") em 10 raízes $(66,7 \%)$, sendo duas fraturas localizadas no terço cervical (20\%), 5 fraturas nos terços médio e apical simultaneamente (50\%) e 3 fraturas nos terços cervical (30\%), também médio e apical simultaneamente. Em cinco raízes não observou-se presença real de fratura radicular (33,3\%).

A presença de traços de fraturas foi visualizada na imagem tomográfica (Figura 1). 

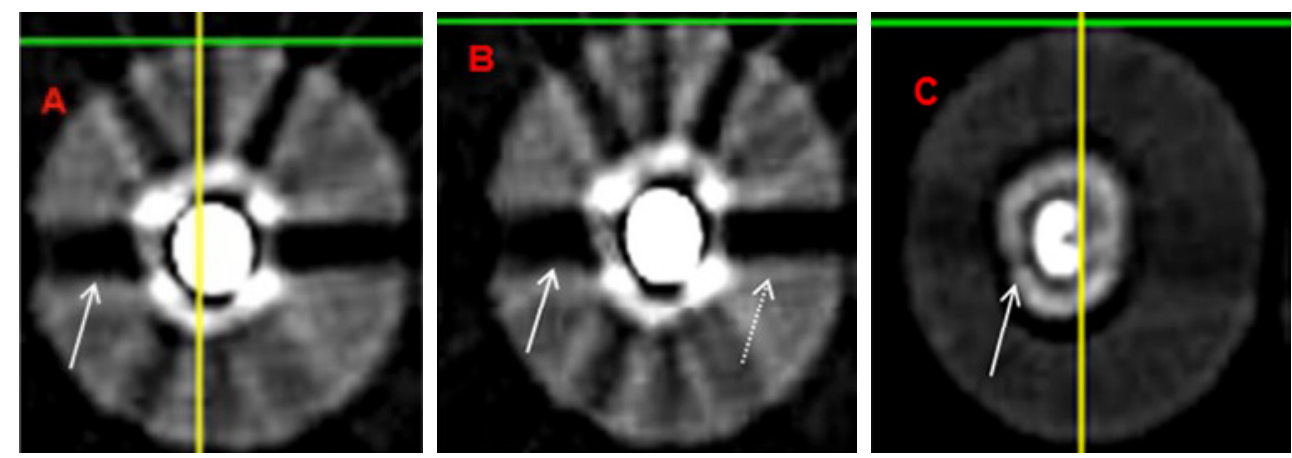

Figura 1 | Imagens tomográficas, em cortes axiais. (A) - presença de beam hardening tipo streak (seta) em imagem antes da indução da fratura. (B) - presença de beam hardening tipo streak (seta) e cupping (seta tracejada). (C) - presença de traço de fratura em terço cervical (seta).

Quanto à presença de fratura no momento inicial $\left(\mathrm{T}_{\mathrm{o}}\right)$, onde não há presença de fraturas, observamos que o avaliador 1 identificou $23 \%$ das raízes como fraturadas, sendo $66,7 \%$ das fraturas visualizadas no terço apical, e 33,3\% no terço médio. Já o avaliador 2 identificou $47 \%$ como fraturados, sendo $57,1 \%$ das fraturas visualizadas nos terços cervical e médio, $28,6 \%$ visualizadas no terço médio e $14,3 \%$ no terço apical (Gráfico 1). No momento final ( $\left.\mathrm{T}_{1}\right)$, após os dentes terem sido submetidos à fratura, observamos que o avaliador 1 identificou $70 \%$ das raízes como fraturadas, sendo $40 \%$ das fraturas visualizadas no terço médio e apical, $20 \%$ no terço médio, $20 \%$ no terço cervical e médio, 10\% no terço apical e 10\% nos terços cervical, médio e apical simultaneamente. Já o avaliador 2 identificou $63 \%$ como fraturadas, sendo 44,4\% no terço médio e apical, $22,2 \%$ no terço apical, 11,1\% no terço cervical, $11,1 \%$ no terço médio e 11,1\% no terço cervical e médio (Gráfico 2). No diagnóstico "verdade", presença real de fratura, verificou-se que $50 \%$ das fraturas estavam no terço cervical e médio, 30\% no terço cervical, médio e apical simultaneamente, e 20\% apenas no terço cervical. Observou-se que nenhuma fratura foi citada no terço cervical e apical simultaneamente (Gráfico 3).

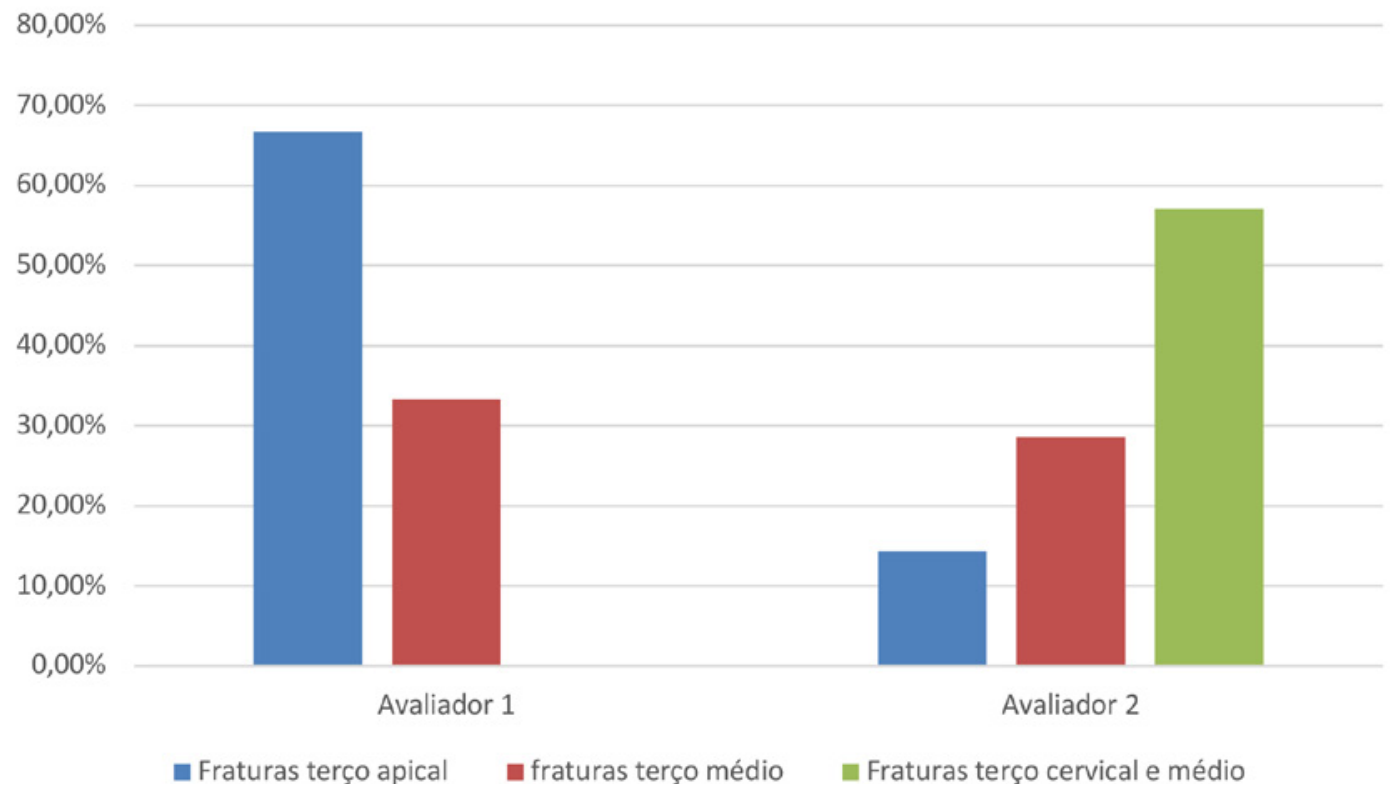

Gráfico 1 | Local das fraturas em $\mathrm{T}_{0}$. 


\section{$100,00 \%$}

$90,00 \%$

$80,00 \%$

$70,00 \%$

$60,00 \%$

$50,00 \%$

$40,00 \%$

$30,00 \%$

$20,00 \%$

$10,00 \%$

$0,00 \%$
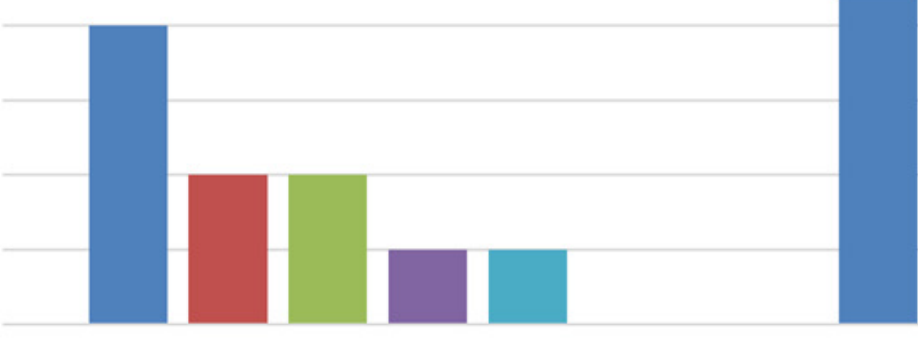

Avaliador 1

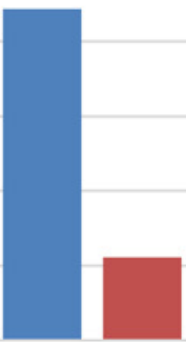

1

\section{avaliador 2}

- Fraturas terço médio e apical

- Fraturas terço médio

Eraturas terço cervical e médio

- Fratura terço apical

= fraturas terços cervical, médio e apical, simultaneamente = Fraturas terço cervical

Gráfico 2 | Local das fraturas em $\mathrm{T}_{1}$.

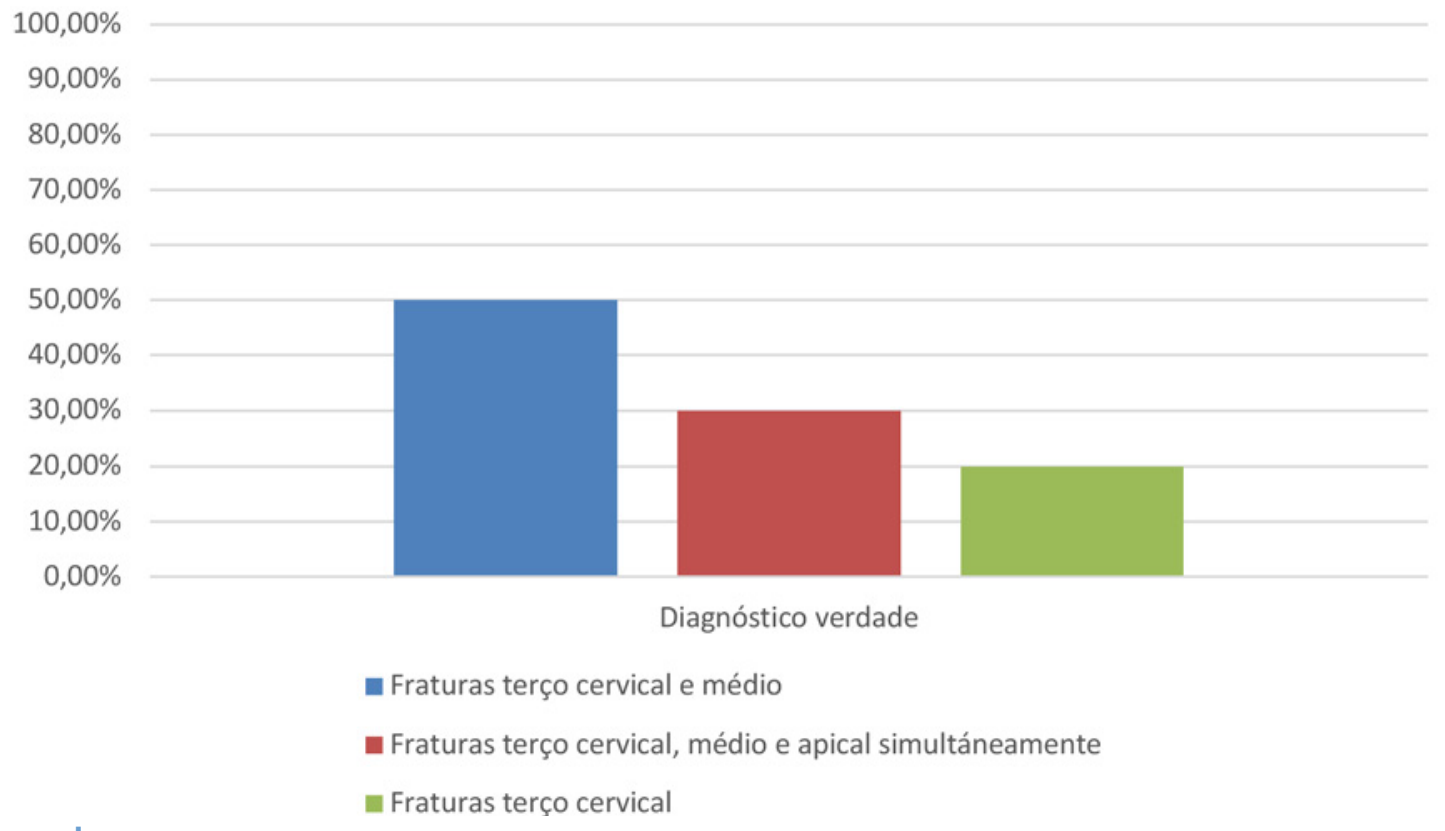

GRÁFICO 3 | Diagnóstico "verdade". 
Ambos avaliadores citaram a presença do artefato beam hardening em $100 \%$ das raízes analisadas.

De acordo com a presença do beam hardening e do corte de visualização das fraturas, observamos que o avaliador 1 no momento inicial $\left(\mathrm{T}_{\mathrm{o}}\right)$ citou $66,7 \%$ da presença de beam hardening no terço médio, enquanto que o avaliador 2 encontrou $60 \%$ no mesmo terço. No momento final $\left(\mathrm{T}_{1}\right)$ os avaliadores encontraram também a maior permanência do artefato beam hardening no terço médio (53,3\%) (Gráfico 4).

Quanto à validade do método diagnóstico por meio da TCFC, medindo a sensibilidade, verificouse que a sensibilidade da tomografia foi de $78 \%$, evidenciando que este método apresenta, portanto, grande validade no diagnóstico de fraturas radiculares. Já a especificidade foi de $63 \%$ e a acurácia de $68 \%$.

Observou-se que a sensibilidade da TCFC foi de $80 \%$ para o avaliador 1 e de $75 \%$ para o avaliador 2, enquanto que a especificidade foi de $70 \% \mathrm{e}$ $55 \%$ respectivamente. Constatou-se ainda que a sensibilidade foi maior que a especificidade no presente estudo (Gráfico 5).

Das 120 amostras, apenas 40 apresentavam presença real de fraturas (diagnóstico "verdade"), pois 60 amostras em $T_{o}$ nunca apresentaram fraturas, e em $\mathrm{T}_{1}$, das 60 imagens ocorreram efetivamente fratura em apenas 40.

Verificou-se concordância, entre os avaliadores, em relação à presença de fraturas em $60 \%$ dos casos (24 dos 40 dentes com fratura real).

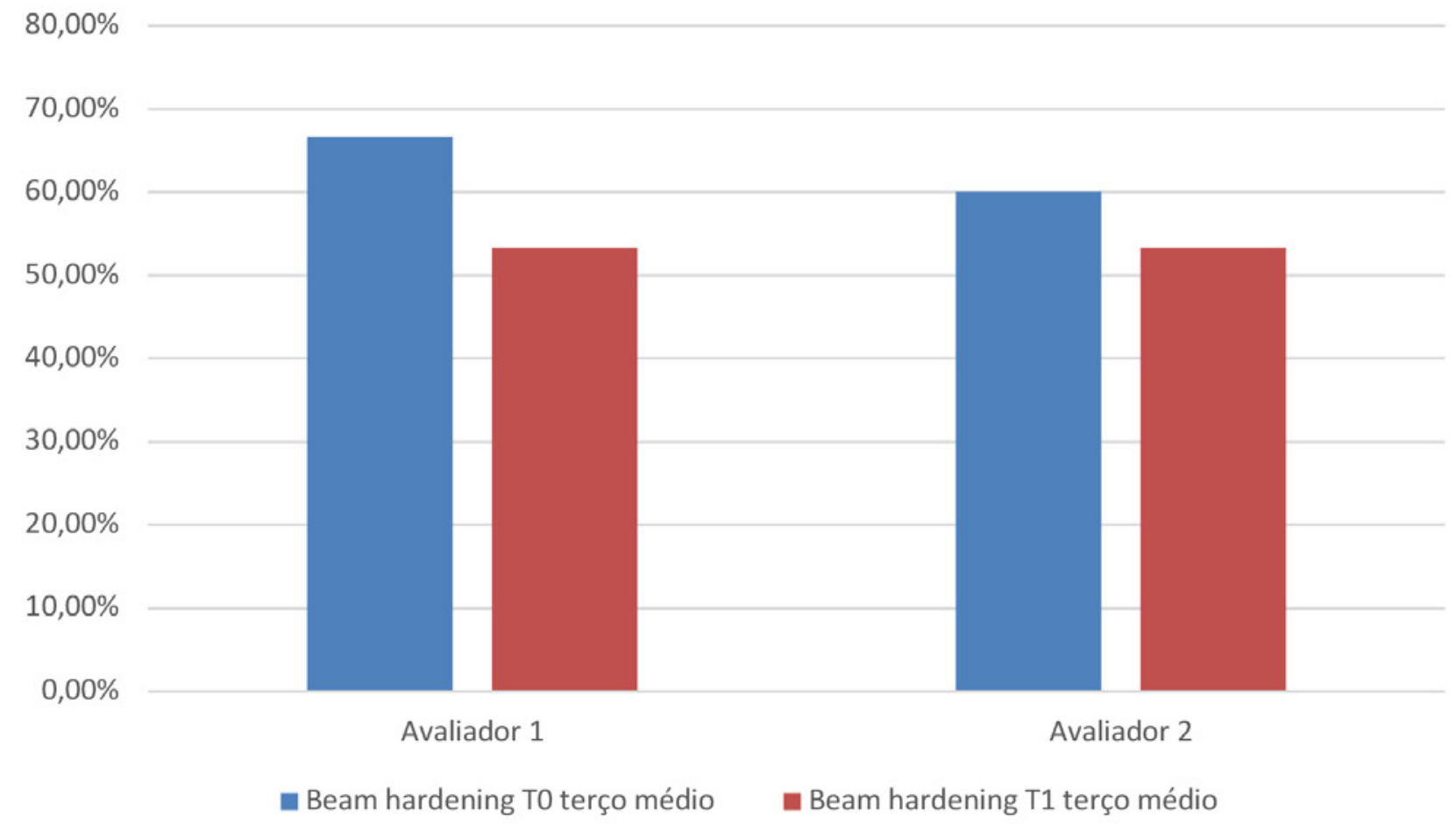

Gráfico 4 | Local do beam hardening em $\mathrm{T}_{0}$ e $\mathrm{T}_{1}$. 


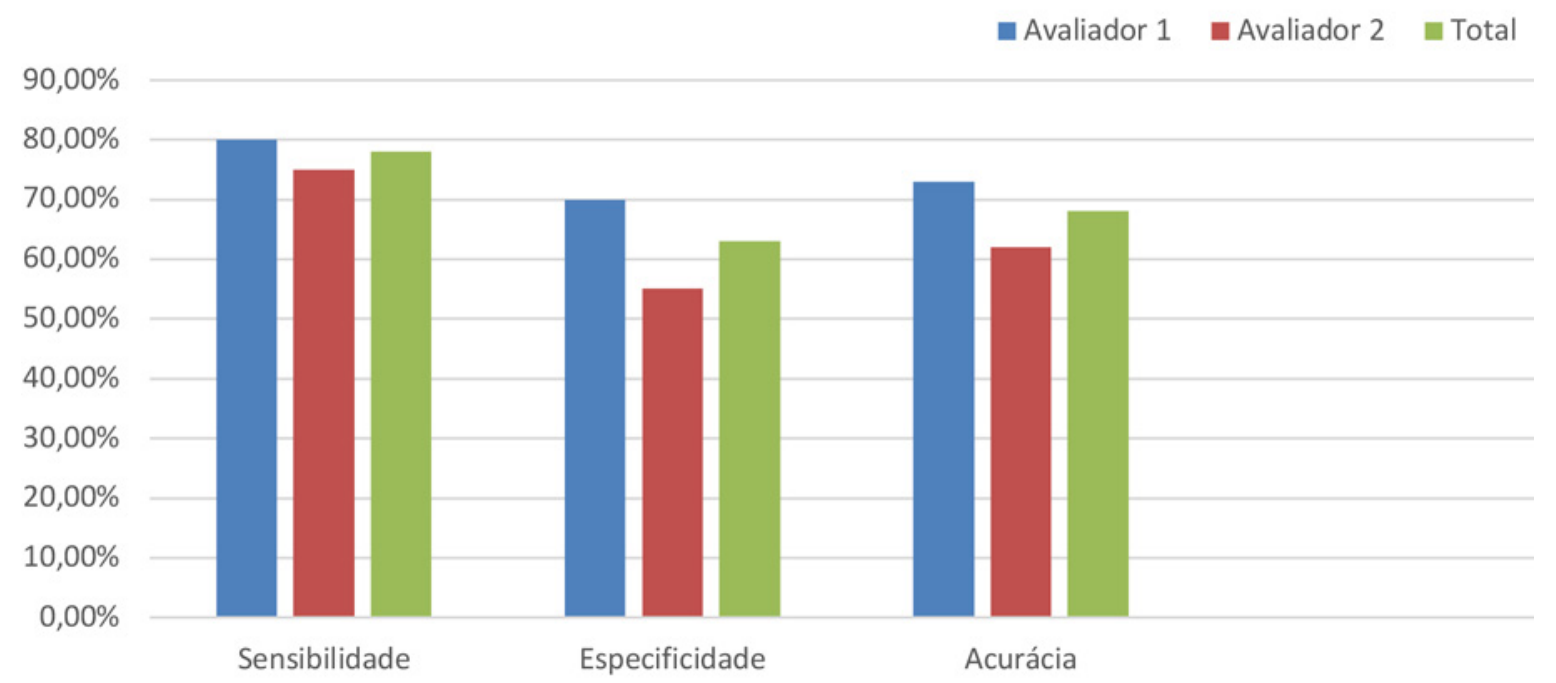

Gráfico 5 | Sensibilidade, especificidade e acurácia da TCFC segundo os avaliadores.

\section{DISCUSSÃO}

Para avaliar a capacidade da TCFC na detecção de FRV foram calculadas as concordâncias intra e interexaminadores, assim como as medidas de acurácia, sensibilidade e especificidade. A concordância intra-examinadores foi moderada para o avaliador $1(0,53)$ e muito baixa para o avaliador $2(0,15)$. Enquanto que a concordância inter-examinadores foi moderada $(0,55)$ para ambos nos dois momentos $\left(\mathrm{T}_{\mathrm{o}}\right.$ e $\left.\mathrm{T}_{1}\right)$. Problemas na reprodutibilidade intra e inter-observadores já foram reportados em outros estudos. ${ }^{5,6,14,15,16,17}$ Outros trabalhos não permitem essa comparação, pois realizaram as avalições com um único observador. ${ }^{18}$ Quando utilizaram vários observadores, estes avaliaram a amostra uma única vez. ${ }^{3,19,20,21,22}$

Apesar dos inúmeros esforços para calibrar os examinadores e assegurar que as imagens fossem vistas de forma padronizada, houve uma série de desacordos entre eles, sugerindo que isso ocorre pela natureza subjetiva da interpretação das imagens, ${ }^{6,23} \mathrm{e}$ pela interferência do beam hardening, causado pela presença do NMF, na detecção da FRV. . $5,5,9,24,25,26,27,28^{2}$

$\mathrm{Em} \mathrm{T}_{\mathrm{o}}$, onde não houve presença de fraturas, $\mathrm{o}$ avaliador 1 indicou 23\% (7 de 30 dentes) dos dentes como fraturados (falso positivos - FP) e o avaliador
2, 47\% FP (14 de 30 dentes). Após as fraturas $\left(\mathrm{T}_{1}\right)$ o avaliador 1 indicou corretamente 64\% (16 de 25 dentes) dos dentes realmente fraturados (verdadeiros positivos - VP), e o avaliador 2 indicou corretamente $60 \%$ (15 de 25 dentes) dos dentes como fraturados (VP). Ressaltamos que os valores de FP foram elevados principalmente em $\mathrm{T}_{1}$. $\mathrm{O}$ que é preocupante, pois pode levar a indicação de exodontia em um dente hígido, sem fratura.

Neste trabalho, após a indução da fratura, pôde-se constatar que as fraturas ocorreram predominantemente nos terços médio e apical, simultaneamente (50\%), sendo consideradas catastróficas ou sem condições de tratamento. ${ }^{29}$ Esse valor aproximou-se dos valores encontrados pelos avaliadores 1 e 2, que foram respectivamente $40 \%$ e $44,4 \%$ das fraturas no terço médio e apical. Em contrapartida, no momento inicial, quando não há presença real de fraturas, o avaliador 1 citou o terço apical com a maior prevalência de fraturas e o avaliador 2 o terço cervical e médio.

Imagens sugestivas de FRV foram visualizadas nos exames tomográficos, diferentemente ao que se observou na inspeção visual em outras regiões das raízes, o que, possivelmente, se pode atribuir, novamente, à presença do artefato beam 
hardening do tipo streak (hipodenso), que simula traços de fratura, levando a um grande número de resultados FP, o que está de acordo com estudos anteriores..$^{5,9,28,30}$

Segundo Patel et al., ${ }^{31}$ as imagens da TCFC não são precisas para a detecção da presença ou ausência da FRV simulada. Chavda et al., ${ }^{6}$ e Neves et al., ${ }^{5}$ concluíram que a TCFC tem limitações significativas para detectar FRV in vivo.

Ambos os avaliadores citaram a presença do artefato beam hardening (do tipo streak) em 100\% das amostras analisadas. Este achado em relação ao NMF está de acordo com o relatado em estudos prévios. ${ }^{5,8,28}$ Tanto o avaliador 1 quanto o avaliador 2, citaram, em ambos os momentos, a presença do beam hardening predominantemente no terço médio, $66,7 \%$ e $60 \%$ respectivamente, o que está de acordo com a literatura de que o beam hardening ocorre predominantemente no término do NMF. ${ }^{23}$

A sensibilidade da TCFC neste estudo foi de o,80 para o avaliador 1, e de 0,75 para o avaliador 2. Enquanto que a especificidade foi de 0,70 para o avaliador 1 e de 0,55 para o avaliador 2, resultando em 0,78 de sensibilidade, 0,63 de especificidade e o,68 de acurácia neste presente estudo. Isto está em concordância com estudos anteriores que demonstraram que a sensibilidade da TCFC varia entre 0,53 e 0,98 enquanto a especificidade varia entre 0,80 e $0,98 .^{20,32,33,34} \mathrm{~A}$ alta sensibilidade da TCFC foi reportada em alguns estudos. . $^{16,19,20,21,35,36,37,38}$ Contudo, os estudos de Patel et al.,31 e Chavda et al., ${ }^{6}$ encontraram maior especificidade para a radiografia periapical em comparação a TCFC.

A presença do artefato beam hardening contribuiu para a diminuição da sensibilidade e da especificidade da TCFC, pelos dados disponíveis e pelas citações em trabalhos anteriores.9,28,30,39 A ocorrência de beam hardening do tipo streak, com formação de linhas hipodensas que simulam traços de fratura em dentes com pinos metálicos, podem ter favorecido o elevado número de resultados FP neste estudo (30/6o leituras). A ocorrência de um número considerável de resultados FP leva-nos a acreditar que houve sim uma interferência do artefato beam hardening da identificação de FRV. Sendo assim, estudos adicionais devem ser conduzidos para esclarecer esses aspectos, já que a ocorrência de diagnóstico FP pode representar a condenação de elementos dentários hígidos.

Deve-se considerar como uma das limitações deste trabalho a utilização de apenas dois examinadores, a grande dificuldade em simular a complexidade da condição in vivo, em que as forças geradas pela mastigação e atividades parafuncionais ocorrem em várias direções, contrariamente às forças unidirecionais dos testes de resistência à fratura utilizados. Ressaltando que a aplicação dos resultados deste estudo para a prática clínica deve ser vista com cautela, e a confecção de coroas unitárias sobre os núcleos coronários é um aspecto que necessita ser incorporado em futuros estudos.

As imagens da TCFC de dentes tratados endodonticamente e com retentores intra-radicular metálicos devem ser cuidadosamente examinados devido a maior densidade dos retentores metálicos e sua capacidade de induzir artefatos de imagens.

Esses artefatos podem afetar o diagnóstico. Assim, estudos futuros deverão avaliar as implicações clínicas dos artefatos metálicos e as estratégias para minimizá-los.

Pelos resultados obtidos neste estudo, podese sugerir que a TCFC constitui um instrumento auxiliar no diagnóstico de FRV em dentes tratados endodonticamente e com NMF. É importante salientar que devido ao grande número de resultados FP e falso negativos (FN) encontrados, esse exame de imagem deve ser apenas mais um método para auxiliar o diagnóstico e que a análise dos sinais clínicos, como alterações no espaço do ligamento periodontal, sensibilidade durante a mastigação, presença de abscesso, perda óssea associada à raiz 
dentária, formação de bolsa periodontal em sítio isolado, além da perda ou mobilidade do retentor intra-radicular ou a mobilidade da coroa, devem ser somados para compor o diagnóstico de possíveis fraturas radiculares.

Deve-se, portanto, associar dados do exame clínico e dos exames por imagem, como a TCFC, para resultar em um diagnóstico mais preciso e acurado, evitando extrações desnecessárias de dentes sem fraturas radiculares.

\section{CONCLUSÕES}

Pode-se concluir neste estudo que a tomografia computadorizada cone beam (TCCB) apresentou $78 \%$ de sensibilidade, $63 \%$ de especificidade e $68 \%$ de acurácia demonstrando ser apenas um método auxiliar no diagnóstico de fraturas radiculares verticais e que o artefato beamhardening (streak) interferiu na detecção das fraturas radiculares verticais, resultando em um número considerável de resultados FP e FN de fraturas.

\section{REFERÊNCIAS}

1. Costa FF, Gaia BF, Umetsubo OS, Pinheiro LR, Tortamano IP, Cavalcanti MG. Use of large-volume cone-beam computed tomography in identification and localization of horizontal root fracture in the presence and absence of intracanal metallic post. J Endod. 2012 Jun;38(6):856-9. doi: 10.1016/j. joen.2012.03.011.

2. Bechara B, McMahan CA, Nasseh I, Geha H, Hayek E, Khawam $\mathrm{G}$ et al. Number of basis images effect on detection of root fractures in endodontically treated teeth using a cone beam computed tomography machine: an in vitro study. Oral Surg Oral Med Oral Pathol Oral Radiol. 2013 May;115(5):67881. doi: 10.1016/j.0ooo.2013.01.026.

3. Safi Y, Ahgdasi MM. Ezoddini-Ardakani F, Beiraghi S, Vasegh Z. Effect of metal artifacts on detection of vertical root fractures using two cone-beam computed tomography systems. Iran Endod J. 2015;10(3):193-8. doi: 10.7508/iej.2015.03.010.

4. Kamburoğlu K, Murat S, Pehlivan SY. The effects of digital image enhancement on the detection of vertical root fracture. Dent Traumatol. 2010 Feb;26(1):47-51. doi: 10.1111/j. 1600-9657.2009.00841.x.
5. Neves FS, Freitas DQ, Campos PS, Ekestubbe A, Lofthag-Hansen S. Evaluation of cone-beam computed tomography in the diagnosis of vertical root fractures: the influence of imaging modes and root canal materials. J Endod. 2014 Oct;40(10):1530-6. doi: 10.1016/j.joen.2014.06.012.

6. Chavda R, Mannocci F, Andiappan M, Patel S. Comparing the in vivo diagnostic accuracy of digital periapical radiography with cone-beam computed tomography for the detection of vertical root fracture. J Endod. 2014 Oct;40(10):1524-9. doi: 10.1016/j.joen.2014.05.011.

7. Hassan B, van der Stelt P, Sanderink G. Accuracy of three-dimensional measurements obtained from cone beam computed tomography surface-rendered images for cephalometric analysis: influence of patient scanning position. Eur J Orthod 2009 Apr;31(2):129-34. doi: 10.1093/ejo/cjno88.

8. Perrella A, Lopes PM, Rocha RG, Fenyo-Pereira M, Cavalcanti MG. Influence of dental metallic artifact from multislice CT in the assessment of simulated mandibular lesions. J Appl Oral Sci. 2010 Mar-Apr;18(2):149-54.

9. Likubo M, Osano T, Sano T, Katsumata A, Ariji E, Kobayashi Ket al. Root canal filling materials spread pattern mimicking root fractures in dental CBCT images. Oral Surg Oral Med Oral Pathol Oral Radiol. 2015 Oct;120(4):521-7. doi: 10.1016/j. oooo.2015.06.030.

10. Zhang Y, Zhang L, Zhu XR, Lee AK, Chambers M, Dong L. Reducing metal artifacts in cone-beam CT images by preprocessing projection data. Int J Radiat Oncol Biol Phys. 2007 Mar;67(3):924-32. doi: 10.1016/j.ijrobp.2006.09.045.

11. Brito-Júnior M, Santos LAN, Faria e Silva AL, Pereira RD, Sousa-Neto MD. Ex vivo evaluation of artifacts mimicking fracture lines on cone-beam computed tomography produced by root different canal sealers. Int Endod J. 2014 Jan;47(1):26-31. doi: 10.1111/iej.12121.

12. Soares CJ, Raposo LH, Soares PV, Santos-Filho PC, Menezes MS, Soares PB, et al. Effect of different cements on the biomechanical behavior of teeth restored with cast dowel-and-cores - in vitro and FEA analysis. J Prosthodont. 2010 Feb;19(2):130-7. doi: 10.1111/j.1532-849X.2009.00527.x.

13. Lopes HP, Siqueira Junior JF, Elias CN. Preparo químico-mecânico dos canais radiculares. In: Lopes HP, Siqueira Junior JF, Elias CN. Endodontia: biologia e técnica. 2a ed. Rio de Janeiro: Guanabara Koogan; 2004. p. 419-80

14. Tsesis I, Kamburoğlu K, Katz A, Tamse A, Kaffe I, Kfir A. Comparison of digital with conventional radiography in detection of vertical root fractures in endodontically treated 
maxillary premolars: an ex vivo study. Oral Surg Oral Med Oral Pathol Oral Radiol Endod. 2008 Jul;106(1):124-8. doi: 10.1016/j.tripleo.2007.09.007.

15. Kamburoğlu K, Murat S, Yüksel SP, Cebeci ARI, Horasan S. Detection of vertical root fracture using cone-beam computerized tomography: an in vivo assessment. Oral Surg Oral Med Oral Pathol Oral Radiol Endod. 2010 Feb;109(2):e74-81. doi: 10.1016/j.tripleo.2009.09.005.

16. Da Silveira PF, Vizzotto MB, Liedke GS. da Silveira HL, Montagner F, da Silveira HE. Detection of vertical root fractures by digital radiographic examination and cone beam computed tomography - an in vitro analysis. Dent Traumatol. 2013 Feb;29(1):41-6. doi: 10.1111/j. 1600-9657.2012.01126.x.

17. European Society of Endodontology, Patel S, Durack C, Abella F, Roig M, Shemesh H, et al. European Society of Endodontically position statement: the use of CBCT in endodontics. Int Endod J. 2014 Jun;47(6):502-4. doi: 10.1111/iej.12267.

18. Kositbowornchai S, Nuansakul R, Sikram S, Sinahawattana S, Saengmontri S. Root fracture detection: a comparison of direct digital radiography with conventional radiography. Dentomaxillofac Radiol. 2001 Mar;30(2):106-9. doi: 10.1038/ $\mathrm{sj} / \mathrm{dmfr} / 4600587$.

19. Hannig C, Dullin C, Hülsmann M, Heidrich G. Three-dimensional, non-destructive visualization of vertical root fractures using flat panel volume detector computer tomography: an ex vivo in vitro case report. Int Endod J. 2005 Dec;38(12):904913. doi: 10.1111/j.1365-2591.2005.01033.x.

20. Mora MA, Mol A, Tyndall DA, Rivera EM. In vitro assessment of local computed tomography for the detection of longitudinal tooth fractures. Oral Surg Oral Med Oral Pathol Oral Radiol Endod. 2007 Jun;103(6):825-9.

21. Kamburoğlu K, Cebeci ARI, Gröndahl HG. Effectiveness of limited cone-beam computed tomography in the detection of horizontal root fracture. Dent Traumatol. 2009 Jun;25(3):256-61. doi: 10.1111/j.1600-9657.2009.00770.x.

22. Ozer SY. Detection of vertical root fractures by using cone beam computed tomography with variable voxel sizes in an in vitro model. J Endod. 2011 Jan;37(1):75-9. doi: 10.1016/j. joen.2010.04.021.

23. Leader DM. CBCT is valuable for diagnosis of tooth fracture. Evid based Dent. 2014 Mar;16(1):23-4. doi: 10.1038/ sj.ebd.6401082.

24. Suojanen JN, Regan F. Spiral CT Scanning of the Paranasal Sinuses. AJNR Am J Neuroradiol. 1995 Apr;16(4):787-9.
25. Katsumata A, Hirukawa A, Noujeim M, Okumara S, Naitoh m, Fujishita M, Ariji E, Langlais RP. Image artifact in dental cone-beam CT. Oral Surg Oral Med Oral Pathol Oral Radiol Endod. 2006 May;101(5):652-7. doi: 10.1016/j.tripleo.2005.07.027.

26. Loubele M, Jacobs R, Maes F, Denis K, White S, Coudyzer $\mathrm{W}$, et al. Image quality vs radiation dose of four cone beam computed tomography scanners. Dentomaxillofac Radiol. 2008 Sep;37(6):309-18. doi: 10.1259/dmfr/16770531.

27. Scarfe WC, Farman AG. What is cone-beam CT and how does it work? Dent Clin North Am. 2008 Oct;52(4):707-30. doi: 10.1016/j.cden.2008.05.005.

28. Jakobson SJM, Westphalen VPD, Silva Neto UX, Fariniuk LF, Schroeder AGD, Carneiro E. The influence of metallic posts in the detection of vertical root fractures using different imaging examinations. Dentomaxillofac Radiol. 2014;43(1):20130287. doi: 10.1259/dmfr.20130287.

29. Cormier CJ, Burns DR, Moon P. In vitro comparison of the fracture resistance and failure mode of fiber, ceramic and conventional post systems at various stages of restoration. $\mathrm{J}$ Prosthodont. 2001 Mar;10(1):26-36.

30. Corbella S, Taschieri S, Samaranayake L, Tsesis I, Nemcovsky C, Del Fabbro M. Implant treatment choice after extraction of a vertically fractured tooth: a proposal for a clinical classification of bony defects based on a systematic review of literature. Clin Oral Implants Res. 2014 Aug;25(8):946-56. doi: 10.1111/clr.12164.

31. Patel S, Brady E, Wilson R, Brown J, Mannocci F. The detection of vertical root fractures in root filled teeth with periapical radiographs and CBCT scans. Int Endod J 2013 Dec;46(12):1140-52. doi: 10.1111/iej.12109.

32. Wang P, Yan XB, Lui DG, Zhang WL, Zhang Y, Ma XC. Detection of dental root fractures by using cone-beam computed tomography. Dentomaxillofac Radiol. 2011 Jul;40(5):290-8. doi: $10.1259 / \mathrm{dmfr} / 84907460$.

33. Metska ME, Aartman IH, Wesselink PR, Özok AR. Detection of vertical root fracture in vivo in endodontically treated teeth by cone beam computed tomography scans. J Endod. 2012 Oct;38(10):1344-7. doi: 10.1016/j.joen.2012.05.003.

34. Makeeva IM. Byakova SF, Novozhilova NE, Adzhieva, Golubeva GI, Grachev K, et al. Detection of artificially induced vertical root fractures of different widths by cone beam computed tomography in vitro and in vivo. Int Endod J. 2016 Oct;49(10):980-9. doi: 10.1111/iej.12549.

35. Youssefzadeh S, Gahleitner A, Dorffner R, Bernhart T, Kainberger FM. Dental vertical root fractures: value of CT in detection. Radiology. 1999 Feb;210(2):545-9.

36. Bernardes RA, de Moraes IG, Duarte MAH, Azevedo BC, de Azevedo JR, Bramante CM. Use of cone-beam volumetric 
tomography in the diagnosis of root fractures. Oral Surg Oral Med Oral Pathol Oral Radiol Endod. 2009 Aug;108(2):270-7. doi: 10.1016/j.tripleo.2009.01.017.

37. Wenzel A, Haiter-Neto F, Frydenberg M, Kirkevang LL. Variable-resolution cone-beam computerized tomography with enhancement filtration compared with intraoral photostimulable phosphor radiography in detection of transverse root fractures in an in vitro model. Oral Surg Oral Med Oral Pathol Oral Radiol Endod. 2009 Dec;108(6):939-45. doi: 10.1016/j.tripleo.2009.07.041.
38. Ozer SY. Detection of vertical root fractures of different thicknesses in endodontically enlarged teeth by cone beam computed tomography versus digital radiography. J Endod. 2010 July;36(7):1245-9. doi: 10.1016/j.joen.2010.03.021.

39. Estrela C, Bueno MR, Silva JA, Porto OCL, Leles CR, Azevedo BC. Effect of intracanal posts on dimensions of cone beam computed tomography images of endodontically treated teeth. Dental Press Endod. 2011 Apr-June;1(1):28-36. doi: 10.14436/2178-3713.1.1.028-036.oar. 